\title{
RETRACTED ARTICLE: Visualized image segmentation for multi-object tracking by weak clustering technique
}

\author{
Liye Gui ${ }^{1} \cdot{\text { Lisha } \mathrm{He}^{1} \cdot \mathrm{Zhongyi} \mathrm{Ni}^{1} \cdot \text { Tianlong Hong }}^{1}$
}

Received: 17 October 2017 / Revised: 28 October 2017 / Accepted: 3 November 2017 /

Published online: 21 February 2018

(C) Springer Science+Business Media, LLC, part of Springer Nature 2018

The Editor-in-Chief has retracted this article [1], which was published as part of special issue "Multi-source Weak Data Management using Big Data", because its content has been duplicated without permission from unpublished manuscripts authored by R Kishorekumar and $\mathrm{P}$ Deepa, and by Priya Selvam and Santhi Balachandran. In addition, there is evidence suggesting authorship manipulation and an attempt to subvert the peer review process.

The authors have not responded to correspondence about this retraction.

\section{References}

1. Gui, L., He, L., Ni, Z. et al. Visualized image segmentation for multi-object tracking by weak clustering technique. Multimed Tools Appl (2018). https://doi.org/10.1007/s11042017-5392-4

Electronic supplementary material The online version of this article (https://doi.org/10.1007/s11042-0175392-4) contains supplementary material, which is available to authorized users.

\section{Liye Gui}

guiliye1778@126.com

1 School of Aeronautics and Astronautics, Zhejiang University, Hangzhou, China 